\title{
Uma câmara de coleóptero (Coprinisphaera) do Cretáceo Superior, Bacia Bauru
}

\author{
Ismar de Souza Carvalho', Diego Evan Gracioso ${ }^{2}$ \& Antonio Carlos Sequeira Fernandes ${ }^{3}$
}

\begin{abstract}
Resumo Na Formação Adamantina (Bacia Bauru, Turoniano-Santoniano) foi identificado o icnogênero Coprinisphaera, sendo o exemplar atribuído à icnoespécie Coprinisphaera cf. C. ecuadoriensis Sauer, 1955. Nos ambientes continentais, as bioturbações podem ser produzidas por diferentes grupos de invertebrados, tais como os nemátodes, anelídeos, biválvios, gastrópodes e artrópodes. As rochas da Formação Adamantina compreendem uma sucessão de arenitos finos, argilitos, siltitos e arenitos argilosos, os quais são interpretados como depositados em barras de canal expostas e planícies de inundação associadas com áreas de ambientes fluviais entrelaçados. Nestes depósitos encontra-se o exemplar atribuído à atividade de coleópteros escarabeídeos, a qual é interpretada como construção para a nidificação desses animais visando a postura e alimentação das larvas, geralmente preservado em paleossolos. A presença de Coprinisphaera na Formação Adamantina contribui com novas informações sobre a distribuição estratigráfica deste icnogênero.
\end{abstract}

Palavras-chave: Coprinisphaera, Bacia Bauru, Cretáceo Superior.

\begin{abstract}
A Coleoptera breeding ball (Coprinisphaera) from the Upper Cretaceous, Bauru Basin, Brazil. In the Adamantina Formation (Bauru Basin, Turonian-Santonian) it was identified Coprinisphaera, attributed to the ichnospecies Coprinisphaera cf. C. ecuadoriensis Sauer, 1955. In the continental environments, the bioturbation can be produced by several groups of invertebrate organisms, including nematodes, annelids, bivalves, gastropods and arthropods. The Adamantina Formation is a sequence of fine sandstones, mudstones, siltstones and muddy sandstones, whose sediments are interpreted as deposited in exposed channel-bars and floodplains areas of braided fluvial environments. In these deposits was found the specimen considered as a breeding ball constructed and provisioned by adult beetles (Scarabeid), to conceal and feed larvae, that are generally preserved in paleosols. The occurrence of Coprinisphaera in the Adamantina Formation allow new data concerning the stratigraphic distribution of this ichnogenus.
\end{abstract}

Keywords: Coprinisphaera, Bauru Basin, Late Cretaceous.

INTRODUÇ̃̃O Normalmente incluídos dentro do icnogênero Coprinisphaera Sauer, 1955, as câmaras de nidificação atribuídas à coleópteros escarabeídeos, popularmente conhecidos como "rola bostas", são conhecidas de várias idades no registro fossilífero cenozóico de diferentes continentes (Genise, 2004). De todas as ocorrências, as da América do Sul, são as consideradas como mais ricas nas icnofaunas de coleópteros (Laza, 2006). Até o momento, os depósitos sul-americanos com essa icnofauna eram conhecidos em formações presentes no Equador, no Uruguai e na Argentina, sendo a mais antiga a Formação Asencio do Uruguai, datada do Eoceno, mas que já chegou a ser considerada anteriormente como de idade cretácica (Genise et al., 2000; Genise, 2004; Laza, 2006).

As rochas onde está presente a ocorrência de uma nidificação de coleóptero pertencem à Formação Adaman- tina, de idade turoniana-santoniana (Castro et al., 1999). Trata-se de uma sequência de arenitos finos, argilitos, siltitos e arenitos argilosos depositados num contexto deposicional de ambiente lacustrino. A porção mais inferior de tais depósitos foi redefinida litoestratigraficamente como Formação Araçatuba por Batezelli et al. (1999).

Durante o intervalo temporal no qual a Formação Adamantina se depositou, o aumento progressivo na aridez, devido à persistência de um clima quente $\mathrm{e}$ altos topográficos circundantes à bacia, conduziu ao desenvolvimento de amplas planícies aluviais, rios entrelaçados e pequenos lagos temporários (Mezzalira, 1980; Campanha et al., 1992; Goldberg \& Garcia, 2000), contexto no qual está inserido o ninho de coleóptero aqui analisado. A ação bioturbadora de insetos em ambientes atuais é também frequente em planícies aluviais e barras arenosas de rios temporários.

1 - Universidade Federal do Rio de Janeiro, Centro de Ciências Matemáticas e da Natureza, Instituto de Geociências, Departamento de Geologia, Rio de Janeiro (RJ), Brasil. Bolsista do CNPq. E-mail: ismar@geologia.ufrj.br

2 - Universidade Federal do Rio de Janeiro, Centro de Ciências Matemáticas e da Natureza, Instituto de Geociências, Departamento de Geologia, Rio de Janeiro (RJ), Brasil. E-mail: evangracioso@ufrj.br

3 - Universidade Federal do Rio de Janeiro, Museu Nacional, Departamento de Geologia e Paleontologia, Rio de Janeiro (RJ), Brasil. Bolsista do CNPq. E-mail: acsfernandes@pq.cnpq.br 
CONTEXTO GEOLÓGICO A Bacia Bauru situase entre as latitudes $18^{\circ} \mathrm{S}$ e $24^{\circ} \mathrm{S}$ e longitudes $47^{\circ} \mathrm{W}$ e $56^{\circ} \mathrm{W}$, distribuindo-se por uma área com cerca de
$370.000 \mathrm{~km}^{2}$ no interior do Brasil, abrangendo os estados de São Paulo, Minas Gerais, Mato Grosso do Sul, Goiás e região nordeste do Paraguai (Fig. 1). Seu pa-

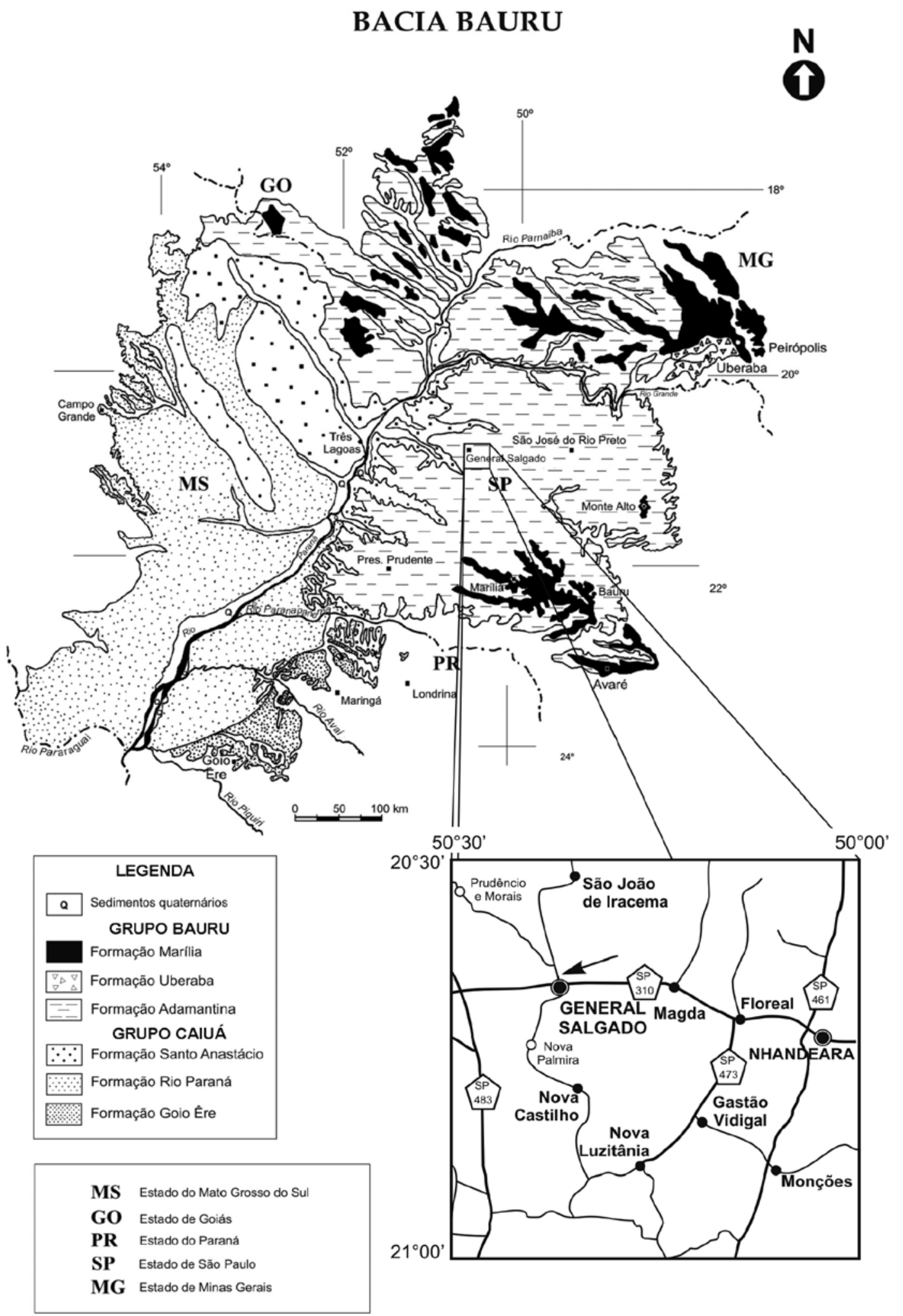

Figura 1 - Mapa litoestratigráfico da Bacia Bauru com a localização do local de ocorrência de Coprinisphaera cf. C. ecuadoriensis Modificado de Fernandes \& Coimbra (1996). 
cote sedimentar é dividido em duas grandes unidades litoestratigráficas: Grupo Caiuá (formações Rio Paraná, Goio Erê e Santo Anastácio) e o Grupo Bauru (formações Adamantina, Uberaba e Marília) (Fernandes, 1998; Dias-Brito et al., 2001).

A Formação Adamantina foi proposta como constituída por arenitos com estratificação cruzada, intercalados com lamitos, siltitos e arenitos lamosos (Soares et al., 1980). De acordo com Fernandes (1998), esta unidade deveria ser subdivida nas formações Vale do Rio do Peixe, São José do Rio Preto e Presidente Prudente. Todavia Paula e Silva (2003) retomou a designação Formação Adamantina por entender que a característica principal dessa unidade é a variabilidade litofaciológica. A Formação Adamantina aflora de modo generalizado em praticamente toda a área de ocorrência do Grupo Bauru, estando recoberta apenas parcialmente pela Formação Marília no compartimento centrooriental da bacia. Em subsuperfície, suas características litológicas são similares às observadas em afloramentos (Paula e Silva et al., 2003), compreendendo depósitos arenosos acanalados, com padrão granulométrico em fining upward e eventuais terminações em sedimentos pelíticos, sugestivos de sedimentação fluvial em canais meandrantes, com deficiência em produtos silto-argilosos (Paula e Silva, 2003; Paula e Silva et al., 2002). Todavia, as interpretações de Fernandes \& Basilici (2009) indicam que os ambientes deposicionais no TuronianoSantoniano da Bacia Bauru refletem condições de clima árido a semi-árido, com a existência de pequenos lagos. Além disso, consideraram que houve marcada oscilação no nível freático atestada por gretas de dessecação e paleossolos drenados. Fernandes \& Basilici (2009) consideraram que este foi um período marcado por fortes mudanças ambientais, representado pela modificação de ambientes de deposição eólicos parcialmente intercalados com fluxos não canalizados em lençóis de areia em ambiente desértico para condições subaquosas em um ambiente lacustre.

Nas proximidades da cidade de General Salgado, na localidade conhecida como Chácara do Negão, situada na margem esquerda da estrada vicinal que liga a cidade de General Salgado (SP) a São José de Iracema a 1,5 km da rodovia SP-310 (Fig. 1), em rochas pertencentes à Formação Adamantina, foi encontrado o exemplar de câmara de coleóptero identificado como pertencente ao icnogênero Coprinisphaera.

Embora já seja conhecida a presença de outros icnofósseis na Formação Adamantina, esta é a primeira ocorrência de Coprinisphaera na formação. Os icnofósseis já conhecidos correspondem a Arenicolites isp., ?Macanopsis isp., Palaeophycus heberti e Taenidium barretti, os quais representam um comportamento escavador de invertebrados endobentônicos. Ocorrem também outras estruturas biogênicas, tais como traços de raízes de plantas, coprólitos e ninhos fossilizados de vertebrados (Fernandes \& Carvalho, 2006). De acordo com estes autores a ocorrência desta associação de icnofósseis sugere o estabelecimento da icnofácies Scoyenia num contexto de planícies de inundação. Ge- ralmente existe uma ampla variedade de icnofósseis de invertebrados nos ambientes continentais, um aspecto observado com frequência por outros autores na análise das icnocenoses não-marinhas (Ratcliffe \& Fagerstrom, 1980; Fitzgerald \& Barrett, 1986; D’Alessandro et al., 1987; Genise et al., 2000). Nos ambientes continentais são reconhecidas três icnofácies associadas aos invertebrados: Coprinisphaera, Scoyenia e Mermia. A icnofácies Coprinisphaera é caracterizada por uma icnodiversidade moderada a relativamente alta originada por estruturas de reprodução de insetos como coleópteros e himenópteros. Tubos com meniscos, escavações de vertebrados e rizólitos também podem ocorrer, caracterizando ecossistemas de comunidades herbáceas terrestres com paleossolos desenvolvidos em uma ampla variedade de ambientes, tais como planícies aluviais, planícies de inundação e depósitos eólicos com vegetação (Genise et al., 2000).

\section{ICNOLOGIA SISTEMÁTICA}

Icnofamília COPRINISPHAERIDAE Genise, 2004

Icnogênero Coprinisphaera Sauer, 1955

Coprinisphaera cf. C. ecuadoriensis Sauer, 1955

(Fig. 2)

Material UFRJ-DG 521-Ic, Departamento de Geologia, Instituto de Geociências, Universidade Federal do Rio de Janeiro.

Descrição Estrutura esférica com cerca de $5,2 \mathrm{~cm}$ de diâmetro externo e $4,3 \mathrm{~cm}$ de altura. Câmara interna isolada, vazia, de contorno cilíndrico e com parede de espessura variável com superfície lisa. A espessura da parede varia de $10 \mathrm{~mm}$ a $21 \mathrm{~mm}$, com $19 \mathrm{~mm}$ de espessura na base, encontrando-se a câmara na parte mais superior da estrutura. Abertura superior com diâmetro de $41 \mathrm{~mm}$, maior que o diâmetro da abertura original, em virtude da má preservação e fragmentação do topo da estrutura. Distribuídas aleatoriamente ao longo da superfície externa da parede ocorrem 21 perfurações de Tombownichnus parabolicus com diâmetros variando de $1,5 \mathrm{~mm}$ a $7,5 \mathrm{~mm}$ e, na superfície da câmara interna, uma perfuração da mesma icnoespécie com diâmetro de 5,0 mm. Não há indicação de existência de câmara secundária (Fig. 2).

Discussão Laza (2006) procedeu a uma revisão do icnogênero Coprinisphaera reconhecendo a existência de cinco icnoespécies: C. ecuadoriensis Sauer, 1955, C. murguiai (Roselli, 1939), C. kraglievichi (Roselli, 1939), C. kheprii Laza, 2006 e C. tonnii Laza, 2006. A primeira, C. ecuadoriensis, icnoespécie tipo do icnogênero, diferencia-se das demais principalmente pela ausência de uma câmara secundária ou de estruturas adicionais circundando a abertura. Entre os principais aspectos diagnósticos que determinam as outras quatro icnoespécies destacam-se: a presença de uma câmara secundária com a forma de um cone invertido e a existência de uma estreita passagem comunicando as duas câmaras em C. murguiai; uma câmara de superfí- 

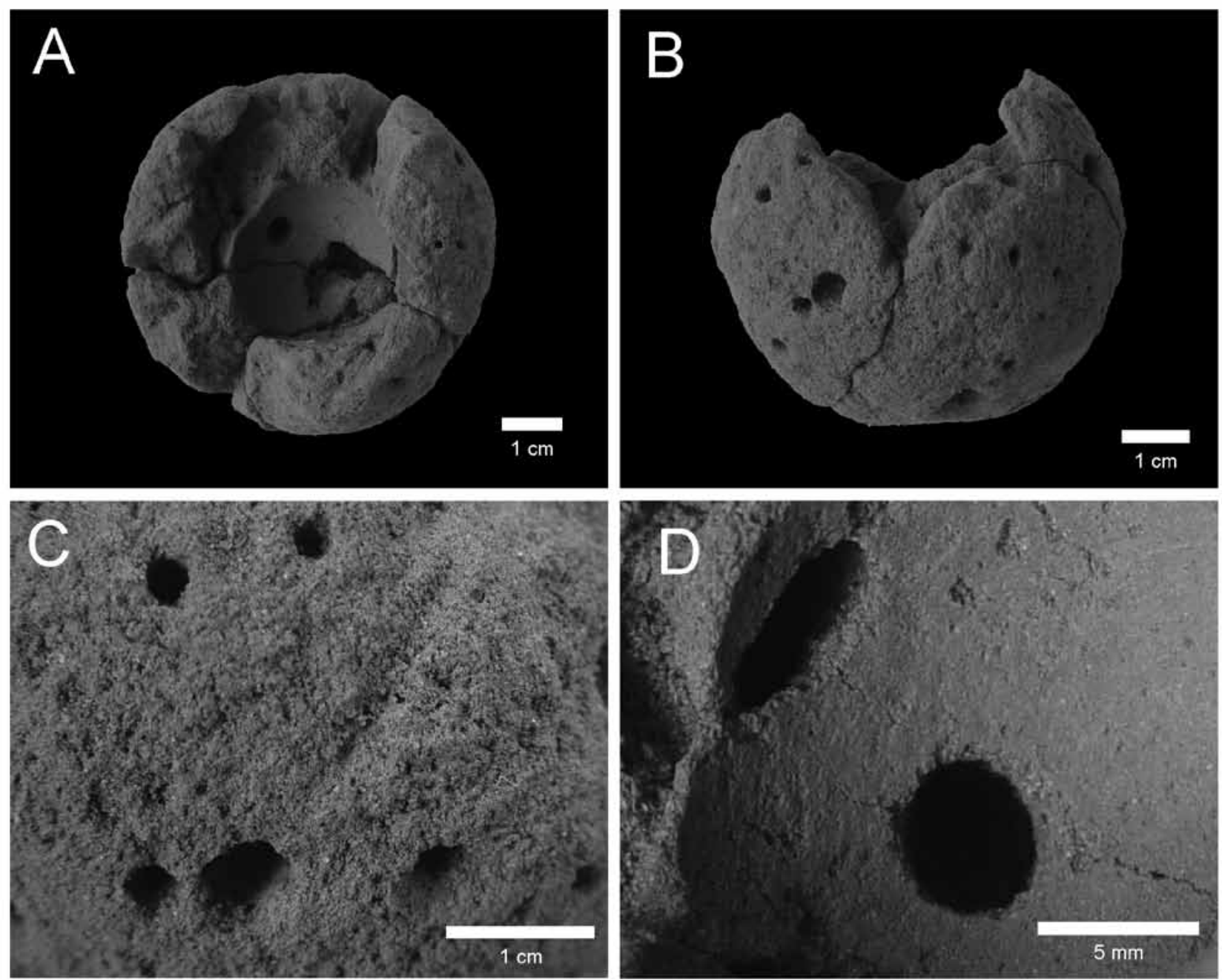

Figura 2 - Coprinisphaera cf. C. ecuadoriensis da Formação Adamantina. (A) Vista superior. (B) Vista lateral. (C) Detalhe das perfurações externas. (D) Detalhe das perfurações internas. Exemplar UFRJ-DG 521-Ic.

cie mais ou menos achatada circundada por uma borda separada da câmara por um canal em C. kraglievichi; presença de uma protuberância em forma de mamilo e abertura alargando-se na base, com câmara interna que pode mostrar contorno irregular, em C. kheprii; e o aspecto de estrutura em forma de pera a biesférica, composta internamente por uma câmara principal conectada a uma câmara secundária menor por uma pequena passagem. As características observadas no exemplar estudado e descritas acima assemelham-se às características diagnósticas de C. ecuadoriensis, diferindo das demais icnoespécies conhecidas de Coprinisphaera.

Contexto ambiental e produtor Coprinisphaera encontra-se entre os icnofósseis mais comuns de paleossolos do cenozoico sul-americano com ocorrências que se distribuem do Paleoceno ao Pleistoceno superior (Buatois et al., 2002; Genise et al., 2000; Genise, 2004). Seu registro mais antigo, entretanto, parecia estar relacionado ao Cretáceo Superior do Uruguai, onde foi assinalado na Formação Asencio e cuja idade é motivo de discussão já que alguns autores a atribuem ao Paleógeno inferior (Genise et al., 2000; Genise \& Bown, 1996). A presença de Coprinisphaera na Formação Adamantina vem, portanto, se constituir num novo marco na distribuição estratigráfica do icnogênero, confirmando seu registro definitivo em rochas de idade cretácica superior representadas por camadas do Turoniano-Santoniano. Apesar de não terem sido registrados fósseis corporais desses animais nas camadas da Formação Adamantina, sua inexistência não poderia ser descartada, já que em muitos casos os icnotáxons de insetos e os fósseis corporais dos produtores potenciais mostram distribuições semelhantes, o que poderia ser o caso dos escarabeídeos. Assim, o Cretáceo não deixou de ser um período crítico durante o qual fósseis corporais como os de coleópteros escarabeídeos são registrados (Genise, 2004).

Normalmente atribuído a bolas de acasalamento construídos por coleópteros escarabeídeos adultos e preservados em paleossolos, este tipo de câmara destinaria-se a conter e alimentar as larvas produzidas 
(Genise \& Cladera, 1995). O icnito resultante, compreendido pelo icnogênero Coprinisphaera, costuma ser associado a ambientes de grandes planícies, como savanas, pradarias ou estepes. Seus produtores normalmente os produzem com os excrementos de vertebrados herbívoros nesses ambientes, havendo uma estreita relação entre esses icnofósseis e paleoambientes herbáceos, habitando principalmente as regiões tropicais a temperadas quentes (Genise et al., 2000). Ambientes de grandes planícies fluviais com vegetação herbácea poderiam assim representar o local de ocorrência dos produtores de Coprinisphaera na Formação Adamantina, onde, ao contrário da presença de mamíferos herbívoros, ocorriam répteis crocodilianos e cujos excrementos fossilizados encontram-se registrados sob a forma de coprólitos bem preservados, provável fonte de matéria-prima para os insetos. Armadillosuchus, Adamantinasuchus e Mariliasuchus, por exemplo, são crocodilomorfos encontrados nessa formação e considerados inclusive com a possibilidade de uma dieta que envolvesse vegetais (Marinho \& Carvalho, 2009; Nobre et al., 2007).

Possíveis produtores Segundo Laza (2006), Coprinisphaera ecuadoriensis assemelha-se às câmaras de nidificação construídas por besouros (Scarabeinae) da tribo Coprini, subtribo Dichotomiina e os da tribo Scarabaeini, subtribo Canthonina, atribuição baseada na condição isolada da câmara, sua forma esférica, espessura da parede e a distribuição moderna destes besouros.

Ocorrências e hábitos dos escarabeídeos fósseis De acordo com Wolf-Schwenninger \& Schawaller (2007) o coleóptero mais antigo assemelhado aos escarabeíde- os é proveniente do Jurássico Inferior da Suiça, sendo também conhecidas ocorrências do Jurássico Superior e do Cretáceo Inferior da Ásia. Os escarabeídeos do Cretáceo Inferior teriam mais provavelmente um hábito xilomicetófago, ao contrário do hábito coprófago, o qual somente é inicialmente conhecido a partir do Cretáceo Superior. Ainda segundo os dois autores, no Brasil, cerca de 14 espécimens fósseis de escarabeídeos foram identificados nas camadas calcárias do Membro Crato da Formação Santana, cuja idade é atribuída ao Cretáceo Inferior; desses exemplares, 11 são provavelmente atribuídos à subfamília Aphodiinae. A presença de $\mathrm{Co}$ prinisphaera na Formação Adamantina, portanto, pode ser uma indicação indireta da existência de representantes de Scarabeinae no Cretáceo Superior do Brasil.

CONCLUSÕES A presença de Coprinisphaera na Formação Adamantina contribui com novas informações sobre a distribuição estratigráfica do icnogênero, anteriormente restrito ao cenozoico. A ocorrência nesta unidade litoestratigráfica permite aumentar a credibilidade de sua presença a partir do Cretáceo Superior, incluindo a representação de sua icnofácies de mesma designação, representativa de paleossolos formados em grandes planícies fluviais de clima quente portadoras de vegetação herbácea.

Agradecimentos Ao Prof. João Tadeu Arruda (General Salgado, Estado de São Paulo) pela cessão da amostra analisada. Este estudo contou com o apoio do Conselho Nacional de Desenvolvimento Científico e Tecnológico $(\mathrm{CNPq})$ e Fundação Carlos Chagas Filho de Amparo à Pesquisa do Estado do Rio de Janeiro (FAPERJ).

\section{Referências}

Batezelli A., Perinotto J.A.J., Etchebehere M.L.C., Fulfaro V.J. \& Saad A.R. 1999. Redefinição litoestratigráfica da unidade Araçatuba e da sua extensão regional na Bacia Bauru, Estado de São Paulo, Brasil. In: UNESP, Simp. Cretáceo do Brasil, 5, Boletim, p. 195-200.

Buatois L., Mángano M., Aceñolaza F. 2002. Trazas fósiles: señales de comportamiento en el registro estratigráfico. Museo Paleontológico Egidio Feruglio, Trelew, 382 p.

Campanha V.A., Etchebehere M.L.C., Saad A.R., Fulfaro V.J. 1992. O significado paleogeográfico das novas ocorrências fossilíferas do Grupo Bauru na região do Triângulo Mineiro, MG. In: UNESP, Simp. Bacias Cretácicas do Brasil, 2, Resumos Expandidos, p. 151152.

Castro J.C., Dias-Brito D., Musacchio E.A., Suarez J., Maranhão M.S.A.S., Rodrigues R. 1999. Arcabouço estratigráfico do Grupo Bauru no oeste Paulista. In: UNESP, Simpósio Cretáceo do Brasil, 5, Boletim, p. 509-515.

D’Alessandro A., Ekdale A.A., Picard M.D. 1987. Trace fossils in fluvial deposits of the Duchesne River Formation (Eocene), Uinta Basin, Utah. Palaeogeography,
Palaeoclimatology, Palaeoecology, 61(3/4):285-301.

Dias-Brito D., Musachio E.A., Castro J.C., Maranhão M.S.S., Suárez J.M., Rodrigues R. 2001. Grupo Bauru: uma unidade continental do Cretáceo no Brasil-concepções baseadas em dados micropaleontológicos, isótopos e estratigráficos. Revue Paléobiologique, 20(1):245-304.

Fernandes A.C.S. \& Carvalho I.S. 2006. Invertebrate Ichnofossils from The Adamantina Formation (Bauru Basin, Late Cretaceous), Brazil. Rev. Bras. Paleont., 9(2):211-220.

Fernandes L.A. 1998. Estratigrafia e evolução geológica da parte Oriental da Bacia Bauru (Ks, Brasil). Tese de Doutoramento, Instituto de Geociências, Universidade de São Paulo, 216 p.

Fernandes L.A. \& Basilici G. 2009. Transition of ephemeral palustrine to aeolian deposits in a continental arid semi-arid environment (Upper Cretaceous Bauru Basin, Brazil) Cretaceous Research, 30:605-61.

Fernandes L.A. \& Coimbra A.M. 1996. A bacia Bauru (Cretáceo Superior, Brasil). An. Acad. Bras. Ciênc., 68:195-205.

Fitzgerald P.G. \& Barret P.J. 1986. Skolithos in a Permian 
braided river deposit, Southern Victoria Land, Antarctica. Palaeogeography, Palaeoclimatology, Palaeoecology, 52:237-247.

Genise J.F. 2004. Ichnotaxonomy and ichnostratigraphy of chambered trace fossils in palaeosols attributed to coleopterans, ants and termites. Geol. Soc. London, Sp. Public., 228:419-453.

Genise J.F. \& Cladera G. 1995. Application of computerized tomography to study insect traces. Ichnos, 4:77-81.

Genise J.F. \& Bown T.M. 1996. Uruguay Roselli 1938 and Roselichnus, n. ichnogenus: two ichnogenera for cluster of fossil bee cells. Ichnos, 4:199-217.

Genise J.F., Mángano M.G., Buatois L.A., Laza J.H., Verde M. 2000. Insect trace fossil associations in paleosols: the Coprinisphaera Ichnofacies. Palaios, 15:49-64.

Goldberg K. \& Garcia A.J.V. 2000. Palaeobiogeography of the Bauru Group, a dinosaur-bearing Cretaceous unit, northeastern Paraná Basin, Brazil. Cretaceous Research, 21:241-254.

Laza J.H. 2006. Dung-beetle fossil brood balls: the icnhnogenera Coprinisphaera Sauer and Quirogaichnus (Coprinisphaeridae). Ichnos, 13:217-235.

Marinho T.S. \& Carvalho I.S. 2009. An armadillo-like sphagesaurid crocodyliform from the Late Cretaceous of Brazil. Jour. South Am. Earth Sci., 27:36-41.

Mezzalira S. (1980) Aspectos paleoecológicos da Formação Bauru. In: Mesa Redonda: A Formação Bauru no Estado de São Paulo e regiões adjacentes. Publ. Esp. Soc. Bras. Geol., 7:1-9.

Mikulás R. \& Genise J.F. 2004. Traces within traces: holes, pits and galleries in walls and fillings of insect trace fossils in paleosols. Geologica Acta, 1(4):339-348.

Nobre H.N., Carvalho I.S., Vasconcellos F.M., Souto P.R. 2007. Feeding behavior of the Gondwanic
Crocodylomorpha Mariliasuchus amarali from the Upper Cretaceous Bauru Basin, Brazil. Gondwana Research, 11:1-21.

Paula e Silva F. 2003. Geologia de subsuperfície e hidroestratigrafia do Grupo Bauru no Estado de São Paulo. Tese de Doutoramento, Instituto de Geociências e Ciências Exatas, Universidade Estadual Paulista, 166 p.

Paula e Silva F., Chang H.K., Caetano-Chang M.R. 2002. Caracterização das unidades neocretáceas da Bacia do Paraná na região de São José do Rio Preto (SP) com base na análise de perfis geofísicos e suas implicações estratigráficas. In: UNESP, Simp. Cretáceo do Brasil, 6, e Simp. sobre el Cretáceo de América del Sur, 2, Boletim, p. 345-349.

Paula e Silva F., Chang H.K. \& Caetano-Chang M.R. 2003. Perfis de referência do Grupo Bauru (K) no Estado de São Paulo. Geoc., 22(número especial):21-32.

Ratcliffe B.C. \& Fagerstrom J.A. 1980. Invertebrate lebensspuren of Holocene floodplains: their morphology, origin and paleoecological significance. Jour. Paleont., 54(3):614-630.

Soares P.C., Landim P.M.B., Fúlfaro V.J., Sobreiro Neto A.F. 1980. Ensaio de caracterização estratigráfica do Cretáceo no Estado de São Paulo: Grupo Bauru. Rev. Bras. Geoc., 10(3):177-185.

Wolf-Schwenninger K. \& Schawaller W. 2007. Coleoptera. In: Martill D.M., Bechly G., Loveridge R.F. (eds.) The Crato fossil beds of Brazil. Cambridge, Cambridge University Press, p. 340-350.

Manuscrito ID 15280

Submetido em 3 de setembro de 2009 Aceito em 25 de novembro de 2009 